\title{
The Effects of Extravehicular Activity (EVA) Glove Pressure on Tactility
}

\author{
Shelby Thompson ${ }^{1}$, Miranda Mesloh ${ }^{1}$, Scott England ${ }^{2}$, \\ Elizabeth Benson ${ }^{2}$, and Sudhakar Rajulu ${ }^{3}$ \\ ${ }^{1}$ Lockheed Martin, JSC, Houston, TX \\ ${ }^{2}$ MEI Technologies, JSC, Houston, TX \\ ${ }^{3}$ Johnson Space Center, NASA, Houston, TX
}

\begin{abstract}
The purpose of the current study was to quantify finger tactility, while wearing a Phase VI Extravehicular Activity (EVA) glove. Subjects were fully suited in an Extravehicular Mobility Unit (EMU) suit. Data was collected under three conditions: bare-handed, gloved at $0 \mathrm{psi}$, and gloved at $4.3 \mathrm{psi}$. In order to test tactility, a series of 30 tactile stimuli (bumps) were created that varied in both height and width. With the hand obscured, subjects applied pressure to each bump until detected tactilely. The amount of force needed to detect each bump was recorded using load cells located under a force-plate. The amount of force needed to detect a bump was positively related to width, but inversely related to height. In addition, as the psi of the glove increased, more force was needed to detect the bump. In terms of application, it was possible to determine the optimal width and height a bump needs to be for a specific amount of force applied for tactility.
\end{abstract}

\section{INTRODUCTION}

As the National Aeronautics and Space Administration (NASA) embarks on its next generation of manned missions that could include such destinations as the moon, Mars or its moons, or even asteroids, it is vital to quantify human performance in terms of requirements for contractors that will build and supply the next generation of space hardware. Central among these pieces of equipment is the Extravehicular Activities suit (EVA suit), particularly the gloves. Second only to vision, properties of the hand such as mobility, strength, and tactility are highly important features of the human-system in terms of performance. Our role in the Habitability and Human Factors Branch at the Johnson Space Center, NASA, is to employ proper human-system integration in order to mitigate risks to mission and crew, while promoting human health and performance. To that end, the study focused on EVA glove performance in order to generate requirements for the next generation glove. In fact, a vital step in human-centered design is the application of these results to designing better humanmachine interfaces.

The current tactility results are part of a larger set of data collected to characterize the Phase VI EVA glove. Other data collected included: hand mobility, tactility, finger and hand strength, dexterity, and functional task performance using EVA tools. Due to the immense amount of data collected, this document will focus only on the tactility results. The goal was to benchmark the Phase VI glove in order to generate requirements that state that the next generation EVA glove must perform as good, or better than, the most current glove. Therefore, it was important that quantifiable and replicable measures be collected to characterize performance.

Tactility is an important issue in the space environment, especially when vision is limited. This is often the case while wearing a space suit, whereby direct viewing of an object is limited by the mobility of the suit, the person within the suit, and the field of view allowed by the helmet. In many cases, astronauts have to rely on touch to find objects in the environment, such as attaching a tether onto the suits D-ring (see Figure 1), or finding the head of a bolt that is out of view.

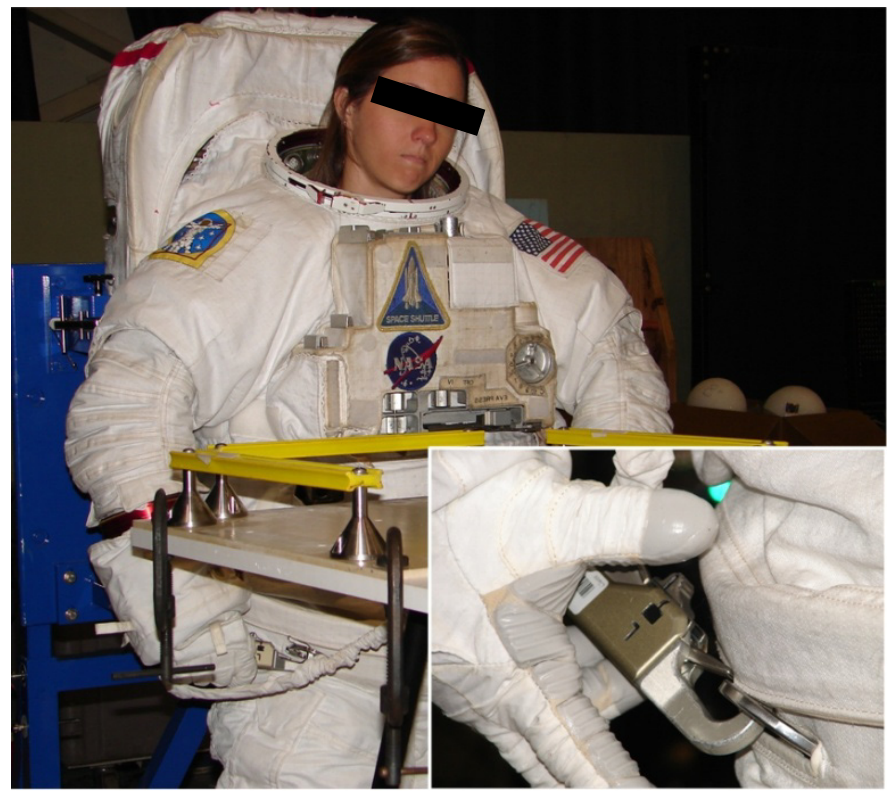

Figure 1. Participant attempting to attach a tether to the D-ring on the suit in the 0psi condition.

One of the major issues in developing a tactility requirement is that traditional tests of tactility are not applicable to space suit gloves. A study of tactile performance with EVA gloves using the two-point discrimination task found the results to be "unreliable and inadequate" (Bishu \& Klute, 1993; Bishu, Klute, \& Kim, 1993). Therefore, in order to test tactility, a series of tactile stimuli (bumps) that varied in height and width was created. These were placed on a force plate, whereby participants pressed on the bumps with only enough force to accurately determine that a bump was present. The resulting force measures were then plotted to determine the range of tactility based upon force needed to detect the bump and size of the bumps. 


\section{METHODS}

\section{Participants}

Eight engineers took part in the glove characteristics study (4 males and 4 females). All were right-handed and represented a range of hand anthropometry based on wrist-to-middle finger tip length, hand width, and middle finger length.

\section{Equipment}

A set of tactile stimuli were milled from a harden resin block using a computer numerical controlled (CNC) machine. The bumps varied over six widths $(2.50,5.00,7.50,10.00,12.50$, $15.00 \mathrm{~mm})$ and five heights $(1.25,2.50,5.00,7.50,10.00 \mathrm{~mm})$ to create 30 bumps. In addition, a no-bump condition was used as a control. The force plate, on to which the bumps were placed, was comprised of 5 MLP-10 force transducers sandwiched between two 0.25 inch aluminum plates. A single transducer was mounted at each corner and in the center of the plates. The five transducers were connected to electronic amplifier modules TMO-1. The amplifiers provided power to the force transducers and also amplified the small electrical signals. The output from the amplifiers was fed in to a Dewetron-5000 data acquisition system with a 16-bit analog capture card. The accuracy from end-to-end of the force plate was $\pm 0.1 \mathrm{lbs}$. This was verified by applying calibrated weights at 3 different locations on the plate. A curtain was used to obscure the participants' vision of the bumps. Figure 2 shows the experimental equipment.

During the evaluation, participants donned an Extravehicular Mobility Unit (EMU), while supported in a donning stand. The Phase VI glove is the first EVA glove to be developed completely with computer aided design (Graziosi, Stein, Ross, $\&$ Kosmo, 2001). This glove is the product of many years of advanced glove research and development combining the lessons learned from the flight program glove designs. The Phase VI glove provides a revolution in EVA glove design and performance by providing better fit for improved crewmember comfort and mobility over earlier model gloves. All participants were properly fitted with a suit and gloves prior to participation.

\section{Procedures}

Each subject was tested under three tactility conditions: barehanded, suited/gloved at $0 \mathrm{psi}$, and suited/gloved at $4.3 \mathrm{psi}$. The barehanded condition can be viewed as a baseline against which the other conditions were examined. In terms of the pressurized conditions, the 0 psi condition reflects what would be experienced during launch and reentry, in which the crew would be suited but little to no pressure would be present. The 4.3 psi condition is nominal pressure for EVA activities.

Each bump was randomly selected from a box and placed upon the force plate. In each condition, the subjects' hand was obscured by a curtain, whereby the test conductor took the index finger and lightly placed it at the tip of the bump. Upon removal of the test conductors' hand, the participant was instructed to press down with only enough force to detect the bump (see Figure 2). In addition, they would respond "yes" if a bump was present and "no" if no bump was detected. There were two no-bump conditions for each set of bumps, resulting in a total of 32 responses. Data is reported in terms of actual force applied (pound-force, lbf).

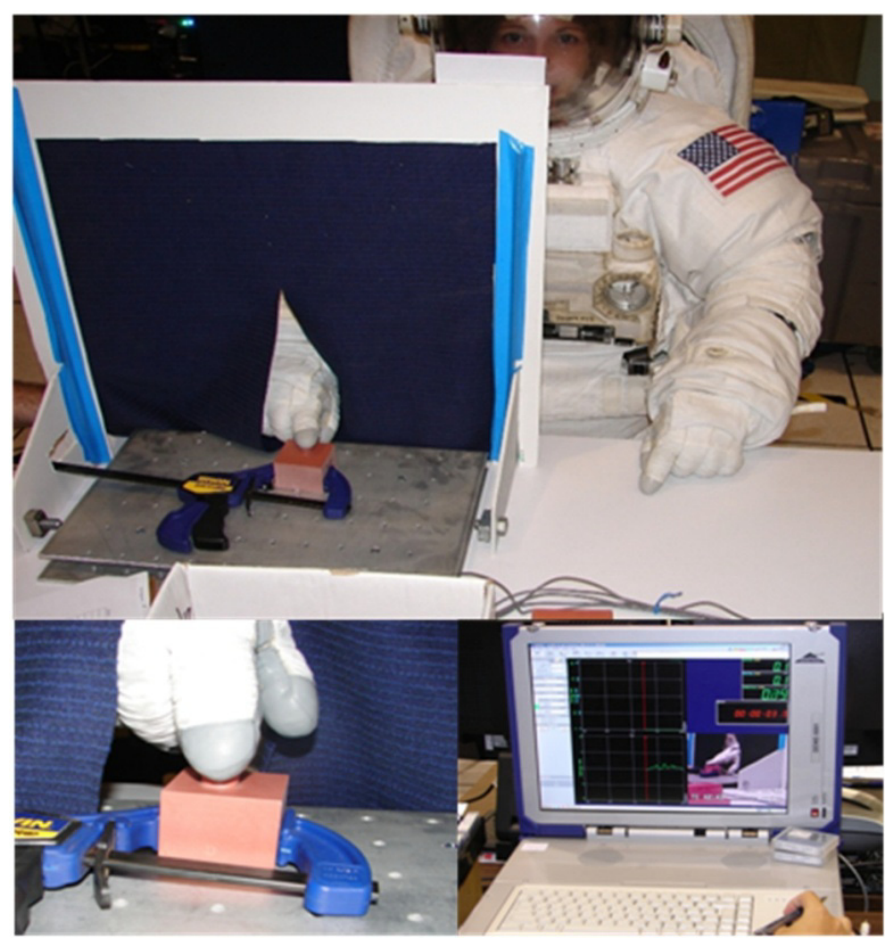

Figure 2. The experimental setup showing the force plate, curtain, and suited participant (top); close-up image of the participant touching the bump (bottom left); and data collection apparatus (bottom right).

\section{RESULTS}

\section{Detection Force}

Indeed, it was possible to quantify tactility for the EVA glove based on the force applied to each bump. In general, the amount of force increased with the glove and pressure. As shown in Table 1, the average force needed to detect the bumps in the barehanded condition was $0.39 \mathrm{lbf}$. However, once gloved this average increased to $3.31 \mathrm{lbf}$, and when pressurized the force increased further to $4.35 \mathrm{lbf}$. The average force and standard deviation for the no-bump conditions were: barehanded $=0.71(0.25), 0$ psi $=3.95(1.07)$, and 4.3 psi $=$ 4.81(0.98).

Table 1.

Force as a Function of Glove Condition

\begin{tabular}{lcccc}
\hline Condition & Min & Max & $M$ & $S D$ \\
\hline Barehanded & 0.24 & 0.90 & 0.39 & 0.14 \\
$0 \mathrm{psi}$ & 2.07 & 4.67 & 3.31 & 0.56 \\
$4.3 \mathrm{psi}$ & 3.13 & 5.18 & 4.35 & 0.53 \\
\hline
\end{tabular}

Note. Force is reported as pounds-force (lbf)

Examination of the ratios revealed that there was a larger increase in the amount of force needed to detect the bump when gloved as compared to barehanded, than for $4.3 \mathrm{psi}$ 
compared to 0 psi (see Figure 3). This suggests that the glove has a greater affect on tactility as compared to increased pressure.

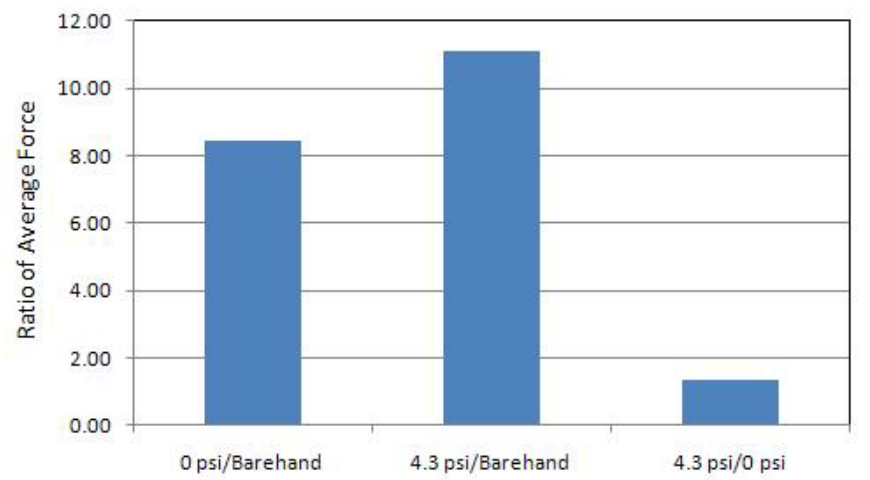

Figure 3. The ratio of average force revealed that the glove had a larger effect on force than pressure.

\section{Effect of Bump Width and Height on Detection}

Early in this process, it was assumed that the width and height of the bumps may have an inverse effect on the amount of force needed to detect the bumps. For example, it seemed logical that while wearing a glove, it may be easier to detect taller bumps versus smaller. Smaller bumps would be lower to the surface of the block, and thus, a greater amount of force maybe needed to accurately distinguish the bump from the surrounding surface with thick EVA gloves on. However, this effect may only reveal itself when the bump is narrow; considering that wider bumps would be more difficult to perceive because the bump would fill the entire surface area of the finger tip, and therefore, participants could not tell the difference between pressing on a flat surface and the tip of a wide bump. Therefore, linear overlay plots were created to examine the independent effects of width and height.

As shown in Figure 4 (top) for the barehanded condition, there was a slight increase in force associated with wider and taller bumps. This same effect becomes more apparent for height with a larger slope in the gloved 0 psi condition, but only slightly increased for width. In the 4.3 psi condition, the slope for width increased as compared to barehanded and 0 psi, but slightly decreased for height. These effects suggest an interaction between width and height, but in addition that pressurization of the glove has a greater effect on width detection.

In terms of making recommendations for tactility, it was our belief that a "sweet spot" could be discovered in which an optimum width and height would be defined as those that took the least amount of force for detection. To that end, a plot of force loadings was created as a function of width and height (see Figure 5).

Going by the most extreme condition, the gloved 4.3 psi, a fairly well defined area of width and height that took minimal force to detect (approximately $<3-3.5 \mathrm{lbf}$ ) can be seen. In addition, this graph also defines the bumps that took a greater amount of force to detect ( $>3.5 \mathrm{lbf}$ ).
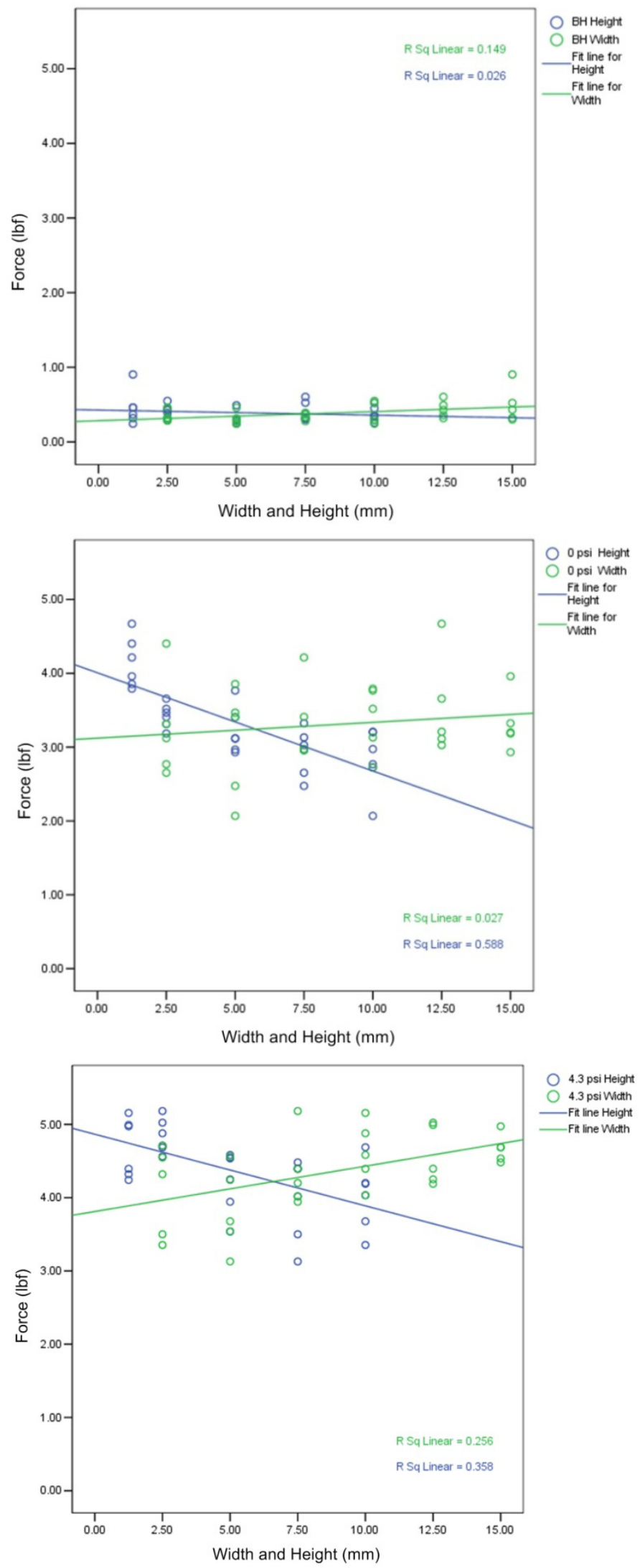

Figure 4. Scatter-overlay plots for force (lbf) as a function of bump width and height. In the barehanded condition (top) there was very little difference in the amount of force needed for detection. However, in the gloved 0 psi condition (center) the amount of force 
dramatically increased for height and only slightly increased for width. In the 4.3 psi condition (bottom), the force for height increased, but width decreased slightly.

\section{CONCLUSIONS}

The primary goal was to develop a method to quantify tactility while wearing an EVA glove in order to develop requirements for the next generation of space gloves. Indeed, this was possible by using pounds of force as a metric to measure tactility. Based on the current results, it can be recommended that objects (e.g., bolt heads, buttons, etc.) be larger than 4.5 $\mathrm{mm}$ in height and smaller than $5 \mathrm{~mm}$ in width. In addition, in order to prevent such errors as accidental actuation (e.g., buttons) the actuation force needs to be greater than $4 \mathrm{lbf}$ (red areas in Figure 5).

The current study also found that width and height interacted with in an inverse fashion. Greater force was needed to detect wider and shorter bumps. This affect was most possibly due to thickness of the gloves, whereby wider bumps were more difficult to detect because the inner surface area of the glove became more evenly distributed across the plump of the finger tip. Furthermore, because of the thickness of the glove layers, taller bumps were more easily detectable.

Additionally, the glove itself had more of an effect on tactility versus pressurizing the glove. In the case of bump height, the addition of pressure appeared to slightly aid detection. This would be logical because once the glove is pressurized it balloons out away from the finger. Therefore, with tall (and narrower) bumps, the participant can detect the inner surface of the gloves contact with the finger tip.

Further studies are planned that will include even smaller and larger percentile hands that were not able to participate due to suit size. In addition, a more complete document that incorporates all test results is planned for later in the year.

\section{REFERENCES}

Bishu, R.R., \& Klute, G. (1993). Investigation of the effects of extravehicular activity (EVA) gloves on performance. $N A S A$ Technical Paper 3401.

Bishu, R.R., Klute, G., \& Kim, B. (1993). The effects of extravehicular activity (EVA) gloves on dexterity and tactility. Proceedings of the Human Factors and Ergonomics Society: $37^{\text {th }}$ Annual Meeting.

Graziosi, D., Stein, J., Ross, A., \& Kosmo, J. (2001). Phase VI advanced EVA glove development and certification for the International Space Station. SAE Technical Paper Series. $31^{\text {st }}$ International Conference on Environmental Systems. Orlando, FL.
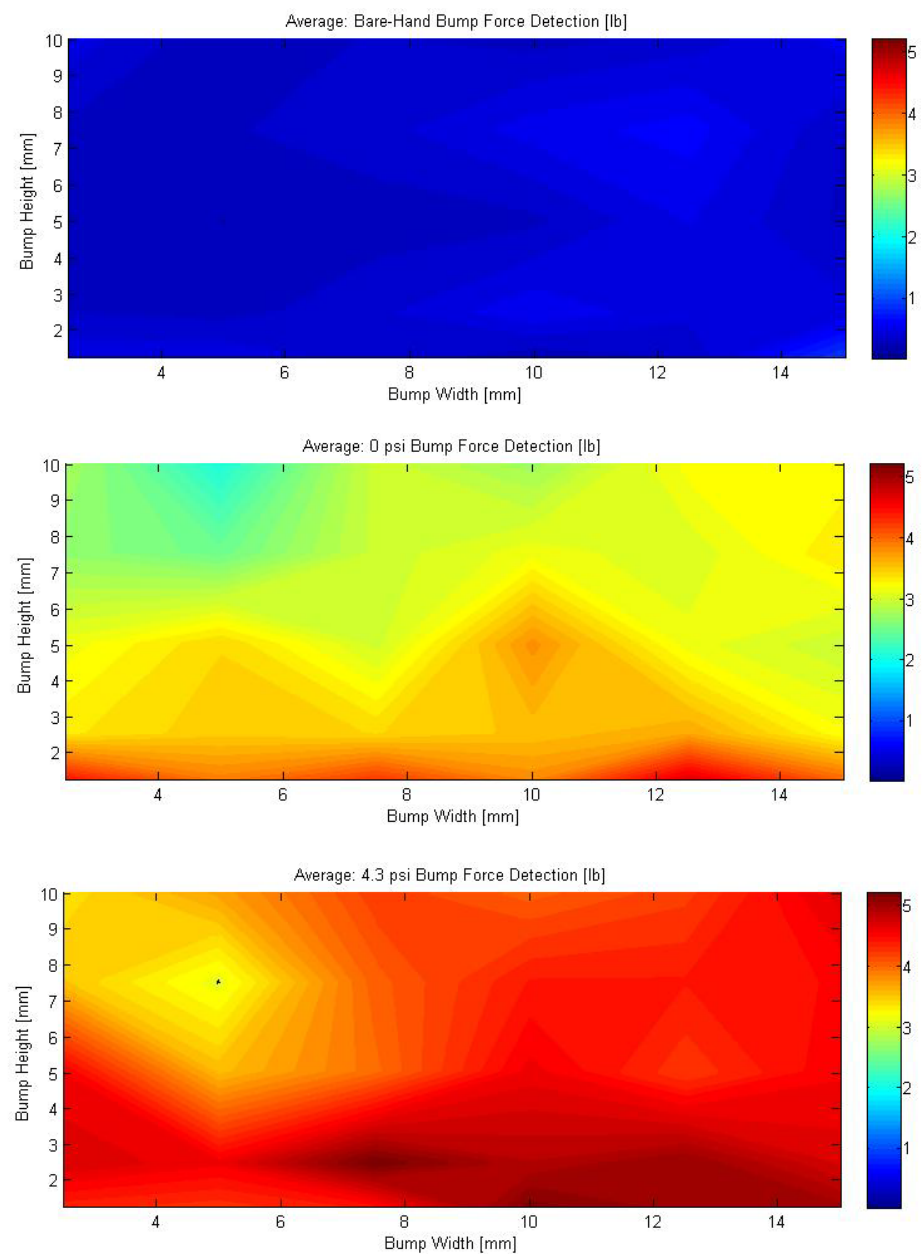

Figure 5. Force-map as a function of bump width (x-axis) and height (y-axis) for the barehanded (top), 0 psi (middle), and 4.3 psi (bottom) conditions. 